\title{
Probing Non-Gaussianity in Confined Diffusion of Nanoparticles
}

Article in Journal of Physical Chemistry Letters · January 2016

DOI: 10.1021/acs.jpclett.5b02624

CITATIONS

6

5 authors, including:

\section{Chundong Xue}

Chinese Academy of Sciences

12 PUBLICATIONS 56 CITATIONS

SEE PROFILE

\section{Guoqing $\mathrm{Hu}$}

Chinese Academy of Sciences

74 PUBLICATIONS 997 CITATIONS

SEE PROFILE
READS

123

\section{Xu Zheng}

Chinese Academy of Sciences

20 PUBLICATIONS 144 CITATIONS

SEE PROFILE

Some of the authors of this publication are also working on these related projects:

Project Enhanced diffusion in complex media View project 


\title{
Probing Non-Gaussianity in Confined Diffusion of Nanoparticles
}

\author{
Chundong Xue, ${ }^{\dagger}$ Xu Zheng, ${ }^{, \dagger}{ }^{\dagger}$ Kaikai Chen, ${ }^{\ddagger}$ Yu Tian, ${ }^{*}$ and Guoqing $\mathrm{Hu}$ \\ †State Key Laboratory of Nonlinear Mechanics, Beijing Key Laboratory of Engineered Construction and Mechanobiology, Institute of \\ Mechanics, Chinese Academy of Sciences, Beijing 100190, China \\ ${ }^{\ddagger}$ State Key Laboratory of Tribology, Tsinghua University, Beijing 100084, China
}

\section{Supporting Information}

ABSTRACT: Confined diffusion is ubiquitous in nature. Ever since the "anomalous yet Brownian" motion was observed, the non-Gaussianity in confined diffusion has been unveiled as an important issue. In this Letter, we experimentally investigate the characteristics and source of non-Gaussian behavior in confined diffusion of nanoparticles suspended in polymer solutions. A time-varied and size-dependent non-Gaussianity is reported based on the non-Gaussian parameter and displacement probability distribution, especially when the nanoparticle's size is smaller than the typical polymer mesh size. This non-Gaussianity does not vanish even at the long-time Brownian stage. By inspecting the displacement autocorrelation, we observe that the nanoparticle-structure interaction, indicated by the anticorrelation, is limited in the short-time stage and makes little contribution to the non-Gaussianity in the long-time stage. The main source of the nonGaussianity can therefore be attributed to hopping diffusion that results in an exponential probability distribution with the large displacements, which may also explain certain processes dominated by rare events in the biological environment.

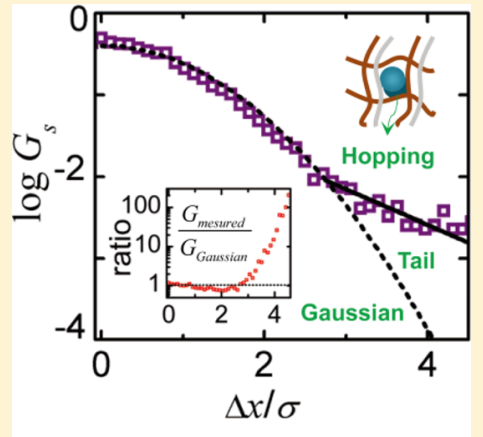

1
$\mathrm{T}$

he diffusive motion of particulate matter (e.g., nanoparticle (NP), protein, and DNA) in a complex medium is of fundamental interest in many interdisciplinary fields, such as biophysics, ${ }^{1-3}$ soft matter, ${ }^{4,5}$ and polymer physics. ${ }^{6-8}$ Different from free Brownian diffusion in simple liquids, the mean squared displacement (MSD) $\left\langle L^{2}(t)\right\rangle$ of the so-called "anomalous diffusion" exhibits a nonlinear relation with elapsed time $t^{9,10}$ i.e., $\left\langle L^{2}(t)\right\rangle \sim t^{\beta}$ where $\beta \neq 1$. $\beta>1$ corresponds to the superdiffusive motion, as observed in the self-motile motion of cells or Janus particles, ${ }^{11,12}$ whereas $\beta<1$ corresponds to the confined and subdiffusive motion, which is suggested to be more ubiquitous in nature. ${ }^{10}$

Previous studies have investigated the typical MSD behavior of confined diffusion, generally showing a transition from a short-time subdiffusion stage restricted locally to a long-time Brownian diffusion stage with linear MSD. ${ }^{13}$ Although the MSD feature can illustrate whether diffusive motion is confined or not, it hardly explains or distinguishes possible mechanisms of confined diffusion. ${ }^{14,15}$ Moreover, as first observed by Granick's group, even Brownian diffusion with linear MSD could be non-Gaussian. ${ }^{15,16}$ This "Brownian but not Gaussian" behavior has attracted much attention because it raises questions of the validity of the frequently invoked Gaussian approximation for describing the diffusive phenomenon in a complex medium. ${ }^{17,18}$ To fully understand this phenomenon, one needs to know the origin and implication of the nonGaussianity. However, the physical source of the nonGaussianity and the influence from the complex environment on the non-Gaussianity remain an open question. ${ }^{19}$ Some theoretical analyses based on stochastic random walk models were found to be incapable of demonstrating the full displacement distribution. ${ }^{20,21}$ The theoretical models considering particle restriction could not predict the features of higher moment of displacement. ${ }^{13,18}$ Other physical influences such as the interaction between particle and surrounding molecules ${ }^{22,23}$ and the dynamics in the heterogeneous structures ${ }^{17,20,24,25}$ were also addressed, yet no consensus has been reached. Thus, the purpose of this study is to identify the physical source of nonGaussianity in confined diffusion and clarify its characteristics.

In this Letter, we study the non-Gaussianity in a model system that contains diffusive polystyrene NPs in poly(ethylene oxide) (PEO) solutions. The motions of NPs were measured using a particle tracking method (details are described in the Supporting Information). The particle tracking method provides detailed displacements and facilitates statistical analysis to quantify non-Gaussianity based on high moment of displacement, displacement probability distribution (DPD), and displacement autocorrelation, which other techniques including dynamic light scattering or fluorescence correlation spectroscopy can hardly provide. ${ }^{26}$ The experiments were performed by controlling the NPs' size (diameter $a=40,100$, and $200 \mathrm{~nm})$, the PEO molecular weight $(\mathrm{MW}=0.6,2,4$, and $8 \mathrm{M})$, and the PEO concentration $(c=0.05-1.5$ wt \%).

Typical results of measured MSD are shown in Figure 1 a. One sees that the MSD behavior varies from a sublinear shorttime stage $(t \sim 10 \mathrm{~ms}$, slope $\beta<1)$ to a linear stage $(t>30 \mathrm{~ms}$, slope $\beta=1)$ in a long-time stage, and the slope on the sublinear stage decreases slightly with increasing PEO concentration. The

Received: November 24, 2015

Accepted: January 19, 2016 


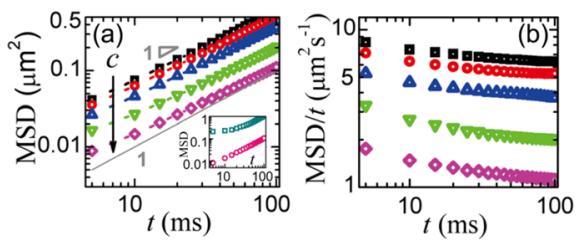

Figure 1. (a) Typical MSD results for $100 \mathrm{~nm}$ NP plotted against time on $\log -\log$ scales at different PEO concentrations, $c=0.05,0.1,0.25$, $0.5,0.75$ wt \%, from top to bottom. Inset: MSD for NPs $a=40$ (cyan rectangulars) and $a=200 \mathrm{~nm}$ (pink circles) in $2 \mathrm{M}$ PEO solution at concentration $c=0.5 \mathrm{wt} \%$. The slope equal to 1 indicates the linear relation between MSD and time, $t$. (b) MSD/time plotted against time to show the transitions from sublinear stage to linear stage.

transition between these two stages is in accordance with previous works. ${ }^{6,7,27,28}$ Figure $1 \mathrm{~b}$ shows the transitions from sublinear stage to linear stage by means of MSD/time. It was believed that the subdiffusion is related to the microscopic viscoelastic response of the flexible PEO molecules. ${ }^{6,27}$ The NPs' motion is slowed by crowded PEO molecules, and this confined effect is enhanced by increasing polymer concentration. The typical time scale of the confined effect could be up to several tens of milliseconds, followed by the long-time stage in which the MSD behavior becomes Brownian because NP diffusion is dominated by macroscopic homogeneous viscosity. $^{29-31}$ In addition, MSD results of particles with different sizes at a fixed PEO concentration are also depicted in the inset of Figure 1a, showing that smaller particles tend to obtain a sublinear stage with a flatter plateau and a longer transition time. Note that the network mesh size, $\xi$, of $c=0.5$ wt $\%$ PEO solution is about $30 \mathrm{~nm}$ while the smallest particle size $a=40$ $\mathrm{nm}$ (see Supporting Information; correlation length $\xi$ is used as the typical mesh size). One explanation is that the comparable particle and mesh sizes make the sublinear stage more remarkable. $^{8,22,32}$

However, there are several important questions that MSD results are unable to answer. First, as explained by Piskorz and Ochab-Marcinek, ${ }^{13}$ all those commonly used models (i.e., elastic force, $27,33,34$ walking confined diffusion with depletion layer, $^{35,36}$ or hopping diffusion ${ }^{8,37,38}$ ) predict the same MSD feature as shown in Figure 1. It is necessary to develop an alternative approach to identify the source of non-Gaussian behavior and unveil the mechanism of the interaction between the NP and surrounding structure. Second, it is still unclear why non-Gaussianity emerges not only in the subdiffusive stage but also in the long-time Brownian stage. The non-Gaussianity needs to be quantified for a better understanding of the anomalous diffusion of NPs in a complex medium.

Therefore, the non-Gaussian parameter, a fourth moment, $\alpha$ $=\left(\left\langle\Delta x^{4}\right\rangle\right) /\left(3\left\langle\Delta x^{2}\right\rangle^{2}\right)-1$, is introduced to quantitatively describe the variation of non-Gaussianity. Generally, $\alpha=0$ indicates a pure Gaussian process such as simple Brownian motion. In the current experiments, however, we obtain positive $\alpha$ which is observed to vary with particle size, elapsed time, PEO concentration, and molecular weight.

Figure 2a,b shows a size-dependent non-Gaussianity, that is, smaller NPs result in a stronger non-Gaussianity regardless of PEO solutions. Explicitly, $\alpha$ is between 0.1 and 0.3 for $40 \mathrm{~nm}$ $\mathrm{NP}$, while $\alpha$ is very close to zero for $200 \mathrm{~nm} \mathrm{NPs}$, indicating that the motion is still Brownian. The measured values of $\alpha$ are consistent with some simulations reported recently. ${ }^{14,39}$ Considering the PEO mesh size is in the order of $50 \mathrm{~nm}$, it is not surprised that a more significant non-Gaussianity will be
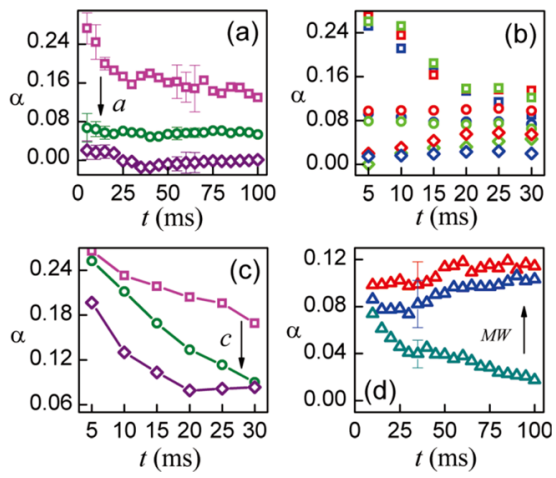

Figure 2. Variation of non-Gaussian parameter $\alpha$ with time: (a) $a=$ 40,100 , and $200 \mathrm{~nm} \mathrm{NPs} \mathrm{(from} \mathrm{top} \mathrm{to} \mathrm{bottom)} \mathrm{in} 2 \mathrm{M}$ PEO solution $(c=0.1 \mathrm{wt} \%)$; (b) three different NPs in $0.5 \mathrm{wt} \% 2 \mathrm{M}$ PEO (blue), 0.5 wt \% $4 \mathrm{M}$ PEO (green), and 0.5 wt $\% 8 \mathrm{M} \mathrm{PEO} \mathrm{(red),} \mathrm{respectively,}$ $a=40,100$, and $200 \mathrm{~nm}$, from top to bottom; (c) $40 \mathrm{~nm} \mathrm{NP}$ in $2 \mathrm{M}$ PEO solution at concentration $c=0.1,0.5$, and $1.5 \mathrm{wt} \%$ (from top to bottom); and (d) $100 \mathrm{~nm} \mathrm{NP}$ in PEO solution with different MWs at a fixed concentration $c=0.5 \mathrm{wt} \%, \mathrm{MW}=8,2$, and $0.6 \mathrm{M}$ (from top to bottom). In panels a and d, error bars are attached based on standard deviations of measured data.

observed for those NPs smaller than the typical size of the surrounding structure. The source of the non-Gaussianity will be analyzed later with the aid of the measured DPD (Figure 3).
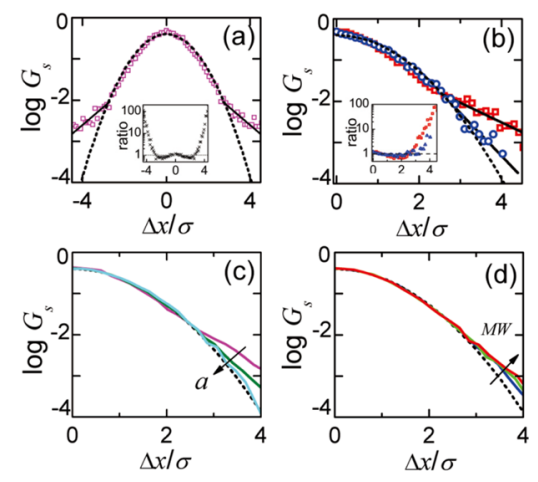

Figure 3. Normalized DPD plotted logarithmically against normalized displacement $\Delta x / \sigma$. Dotted curves represent standard Gaussian distribution. DPD for $40 \mathrm{~nm} \mathrm{NP} \mathrm{(a)} \mathrm{at} t=5 \mathrm{~ms}$ in $2 \mathrm{M}$ PEO solution $(c=0.1 \mathrm{wt} \%)$ and (b) at $t=5$ and $30 \mathrm{~ms}$ in $4 \mathrm{M} \mathrm{PEO}$ solution $(c=0.5 \mathrm{wt} \%)$. In panels a and $b$, the insets show the ratio of observed probability distribution to the Gaussian plotted against normalized displacement. (c) DPD at $t=10 \mathrm{~ms}$ under the conditions corresponding to Figure $2 \mathrm{a}$ and (d) DPD at $t=10 \mathrm{~ms}$ under the conditions corresponding to Figure $2 \mathrm{~d}$.

Moreover, different time-varied tendencies of $\alpha$ are observed. For $40 \mathrm{~nm}$ NPs, $\alpha$ decreases rapidly at short time and then reaches a nearly constant value at about $t \sim 30 \mathrm{~ms}$ (Figure $2 \mathrm{a}, \mathrm{b})$. This time scale agrees with the transition time of the measured MSD shown in Figure 1. For $100 \mathrm{~nm}$ NPs, $\alpha$ remains positive and varies slightly with time; while for $200 \mathrm{~nm} \mathrm{NPs}, \alpha$ is always close to zero, indicating that the non-Gaussianity becomes trivial. More interestingly, the non-Gaussianity of 40 and $100 \mathrm{~nm}$ NPs will not disappear even in the long-time Brownian stage. We notice that some theoretical results suggested the non-Gaussian parameter $\alpha$ would decrease rapidly to zero at the beginning of the long-time Brownian stage. $^{21,24}$ However, the present results show that the non- 
Gaussian behavior could last longer than expected. We underscore the two-stage tendency of $\alpha$ using $40 \mathrm{~nm} \mathrm{NP}$ which suggests the existence of two different mechanisms influencing the non-Gaussian behaviors in short-time or longtime stage. For $100 \mathrm{~nm}$ NPs, the long-time mechanism becomes dominant while the short-time one disappears (Figure $2 a, b)$.

The influence of polymer concentration and MW can be identified from Figure $2 \mathrm{c}$,d. As the mesh size decreases with increasing PEO concentration and becomes smaller than the NP size, the measured $\alpha$ decreases as NP tends to probe the relative homogeneous medium (Figure 2c). The influence of MW is also clearly shown in Figure $2 \mathrm{~d}$ : in short-time stage $(t \sim$ $10 \mathrm{~ms}$ ), the measured $\alpha$ in different PEO solutions are all close to 0.1 , indicating a very weak dependence on MW; but as time increases, $\alpha$ of large MW still remains 0.1 , whereas $\alpha$ of $0.6 \mathrm{M}$ PEO decreases to nearly zero. The effect of MW will be discussed later with the support of the measured DPD (Figure $3 \mathrm{~d})$. These results also reveal the size dependency of the nonGaussian parameter that is consistent with observations in Figure 2a,b.

The DPDs are measured to directly show displacement information on NP's motion (Figure 3). Different from previous studies, ${ }^{4,5,20}$ the standard Gaussian distribution with probability density function $G_{\mathrm{s}}=\frac{1}{\sqrt{2 \pi}} \exp \left[-(\Delta x / \sigma)^{2} / 2\right]$ is introduced based on the normalized displacement $\Delta x / \sigma$, where $\sigma$ is the standard deviation of displacements, which permits a direct comparison between different results. Panels a and $\mathrm{b}$ in Figure 3 show typical DPDs with $40 \mathrm{~nm}$ NPs in 2 and $4 \mathrm{M}$ PEO solutions, respectively. The most obvious deviation compared to standard Gaussian distribution is observed at the tails $(|\Delta x / \sigma|>3)$, showing an approximately one hundred times higher probability of large displacements. The tail distribution is found to decay linearly on the logarithmic plot, implying that the probability function changes to $G_{s} \sim$ $\exp [-(\Delta x / \sigma)]$ (solid lines in Figure 3a,b). The slope of the linear fit varies from approximately -1.1 to -2.4 in Figure $3 b$. Figure $3 \mathrm{c}$ further shows that the tail distribution becomes more pronounced with decreasing NP size. This size dependency indicates the enhanced probability of large displacements when a NP diffuses through the polymer network with a correlation length (defining the average distance between polymer chains) larger than NP size. In the middle part of DPD, a sharper peak at $\Delta x / \sigma=0$ and a narrower shoulder $(|\Delta x / \sigma|=1.5-2.5)$ are visible, representing smaller displacements caused by confinements compared to simple Brownian motion. It is also observed that as time increases the tail probability decreases noticeably (Figure 3b). Recall the corresponding size-dependent and timevaried tendency of $\alpha$ shown in Figure 2; we find that DPD varies with $\alpha$ simultaneously, which suggests that they should have the same origin. Based on the definition of non-Gaussian parameter $\alpha$, i.e., a fourth moment of displacement, the contribution from the tails with large displacements is much more significant than that from the center. Thus, the nonGaussianity depicted by $\alpha$ comes from the enhanced probability of large displacement through the surrounding structures. According to these features, we propose that the source of nonGaussianity is mainly attributed to the hopping diffusion, which means that NPs could hop through the polymer mesh structures nearby intermittently. ${ }^{8,38,40}$ Multiple PEO molecular chains tend to overlap or entangle with each other to form heterogeneous structures like global networks and local compartments in which fast motion of NPs across networks or adjacent compartments would occur. The weak increase of the NP mobility due to nonsticky polymer could also contribute to the long-displacement hop. ${ }^{27}$ This hopping behavior is weakened slightly with increasing time because the hopping distance is restricted by the size of the local heterogeneous structures. However, the long-displacement hop can still be observed in a relatively long time (Figure $3 b$ ), as illustrated by $\alpha$ (Figure 2a,b). Moreover, the influence of PEO MW is not as significant as NP size. But Figure $3 \mathrm{~d}$ still shows that the NP could more easily hop through the network structure of PEO with larger MW. This result also explains the effect of MW on the non-Gaussian parameter shown in Figure $2 \mathrm{~d}$. We propose that the influence of MW can be considered under the theory given by Cai et al. ${ }^{38}$ They suggest that there is a MW-dependent threshold size below which the hopping diffusion becomes easy. When larger MW PEO is used, it becomes easier for the NP whose size is smaller or comparable to the threshold size to hop through the polymer networks. We believe that the divergence between different curves in Figure $2 \mathrm{~d}$ exhibits this threshold behavior, although the influence of MW is weaker than that of NP size, as shown in Figure 3.

The DPD offers an image of how NPs diffuse in the surrounding structures. However, several issues need further consideration. The mechanism of confined diffusion was a longtime debate, and the difficulty that MSD is not able to distinguish different mechanisms was pointed out very recently. ${ }^{13}$ One commonly used simple model assumes that the diffusion is restricted by the elastic force from the nearby polymer molecules. ${ }^{27,34}$ However, it is well-known that the overdamped motion of NPs in a Ornstein-Uhlenbeck process is Gaussian, ${ }^{13}$ which is contradictory to the present observation. Another model based on walking confined diffusion often assumes that there is a depletion layer (containing solvent molecules such as water) between NPs and polymers. ${ }^{35}$ This model can describe some fast diffusion observed in short-time stage (as shown in Figure 1a where short-time MSD presents larger values). Our measured DPD does not support this model either. Theoretically, if the NP's diffusion is much faster inside the depletion layer whereas the outside polymer's motion is relatively immobile, the NP's DPD will quickly equilibrate to a more uniform distribution. Piskorz and Ochab-Marcinek depicted this distribution as plateaulike in the center and diffused to edges. ${ }^{13}$ In contrast, our measured DPD displays a tiny higher peak in the center and a narrower shoulder. Besides the above-mentioned models, hopping diffusion is the only one that can fully depict the measured DPD. Our measured DPD provides evidence for the hopping diffusion whose tail probability decays exponentially and becomes 1-2 orders of magnitude larger than the Gaussian distribution.

As the key point of the non-Gaussianity, ${ }^{17,20}$ the NP's dynamics within the heterogeneous polymer network is another important issue. We thus introduce the displacement autocorrelation to detect the feature of NP's successive displacements $^{11}$ (Figure 4, and see Supporting Information for details). A clear anticorrelation (correlation coefficient $C_{\mathrm{d}}(t)$ $\sim-0.15$ ) around $t=5 \mathrm{~ms}$ is observed. For the results using 4 and $8 \mathrm{M} \mathrm{PEO}$, the anticorrelation remains significant at $t=5$ $\mathrm{ms}$, though the fluctuations increase when $t>10 \mathrm{~ms}$ (inset in Figure 4). The anticorrelation indicates that a large displacement could likely be followed by a small displacement, and vice versa. This is due to the NP-polymer interaction, such as rebound or attraction. ${ }^{21,22}$ In addition, the anticorrelation 


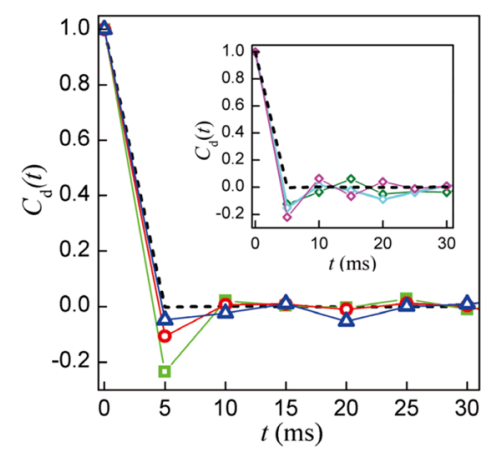

Figure 4. Normalized coefficient of displacement autocorrelation $C_{\mathrm{d}}(t)$ plotted against elapsed time, $t$, for $40 \mathrm{~nm} \mathrm{NP}$ in $2 \mathrm{M}$ PEO solution at concentration $c=0.1 \mathrm{wt} \%$ (blue triangles), $0.5 \mathrm{wt} \%$ (red circles)m and 1.5 wt $\%$ (green squares). Inset: displacement autocorrelation for $40 \mathrm{~nm}$ in $0.5 \mathrm{wt} \% 4 \mathrm{M}$ (olive) and $0.5 \mathrm{wt} \% 8 \mathrm{M}$ (cyan) and $1 \mathrm{wt} \% 8$ M (magenta) PEO. The dashed line represents the displacement autocorrelation of a Gaussian process. Anticorrelation is observed for all conditions around $t=5 \mathrm{~ms}$.

feature becomes more significant as PEO concentration increases. This local effect from the heterogeneous polymer network will disappear at about $t=10 \mathrm{~ms}$, which is approximately in agreement with the transition time scale reported above. Different from some previous theoretical studies that linked the heterogeneous dynamics within local structures as the origin of the non-Gaussianity, ${ }^{17,20,39}$ we observe the influence of this local effect in only the short-time stage. This is in favor of our argument taking hopping diffusion as the main source of non-Gaussianity, because the longdisplacement hop is observed even in the long-time stage. According to Cai et al., ${ }^{38}$ the reason that hopping diffusion can be observed in a long time could be attributed to the slow polymer chain relaxation. This long-displacement hop is vital in many processes, especially where a rare event dominates, ${ }^{16}$ such as catalytic reaction in chemistry and signaling and metabolite shuttling in biology. ${ }^{41}$ The significant increase of the probability value at the non-Gaussian tail offers a signal to evaluate how easily the hopping of DNA or protein occurs. In addition, in contrast to some theoretical studies suggesting diffusivity memory as a key feature of short-time dynamics, ${ }^{20}$ the measured displacement autocorrelation does not show positive correlation between successive motions. Notably, new studies relevant to the interaction between NPs and surrounding structures were performed recently, ${ }^{22,23}$ which is helpful to better understand the physical origin. Furthermore, we noticed that the non-Gaussian behavior was also reported in glassy systems ${ }^{42,43}$ where the non-Gaussian parameter $\alpha$ is up to $1-5$, which is 1 order of magnitude greater than our results. We thus believe that the physical source of non-Gaussianity in soft polymer solutions is not the same as the so-called dynamic heterogeneity in glassy systems. ${ }^{39,44}$ Finally, an explicit mathematic expression of the exponential tail due to hopping diffusion is of great interest, which can characterize the hopping dynamics and should be a function of polymer mesh size, NP mobility, and solution concentration. This interesting issue needs further investigation.

Our results also provide valuable information for the theoretical models based on stochastic random walk processes, ${ }^{10,19,45}$ such as fractional Brownian motion ( $\mathrm{fBm}$ ) or continuous time random walk (CTRW). For DPD, the $\mathrm{BBm}$ models grounded on stationary and Gaussian displacement increment fail to interpret the non-Gaussian distribution. Some CTRW models have difficulty predicting the exponential tail distribution. For the displacement autocorrelation, the $\mathrm{fBm}$ model could be distinguished from unconfined CTRW because the latter does not show anticorrelation feature. ${ }^{20,40}$ Confined CTRW, however, is difficult to distinguish from the $\mathrm{fBm}$ because it exhibits an anticorrelation feature due to boundary reflection. ${ }^{20}$ Theoretical works are ongoing to better illustrate the confined diffusion. ${ }^{21-23}$ It is worth noting that for a validated model the full features of MSD, non-Gaussian parameter $\alpha$, DPD, and displacement autocorrelation, should be simultaneously satisfied.

In summary, this Letter investigates the diffusing behavior of NPs interacting with surrounding polymer chains by means of non-Gaussian parameter $\alpha$, DPD, and displacement autocorrelation. A size-dependent and time-varied non-Gaussianity is observed, especially when the NP's size is smaller than the typical network mesh size. This non-Gaussianity does not vanish even at long-time Brownian stage with linear MSD and thus results in the "anomalous yet Brownian" phenomenon. ${ }^{20}$ An anticorrelation of displacement is observed corresponding to the confinement from surrounding structures in the shorttime stage. We thus propose that the non-Gaussianity could have two sources with different time scales. The main source of non-Gaussianity is connected to the exponential tail distribution with enhanced displacements, which is a clear signal of hopping diffusion. In contrast to the previous studies focusing on the dynamics in the heterogeneous surrounding structures, our results suggest that the effect of local confinement contributes to only short-time non-Gaussianity and becomes trivial in the long-time stage. The present results provide physical insights into the particle-polymer interaction and shed light on the debate of the physical origin of NP dynamics in soft-matter systems.

\section{ASSOCIATED CONTENT}

\section{S Supporting Information}

The Supporting Information is available free of charge on the ACS Publications website at DOI: 10.1021/acs.jpclett.5b02624.

Experimental details, including sample preparation, experimental observation, image processing, statistical analysis of NP motion, and the estimation of PEO mesh size (PDF)

Raw experimental video (see Supporting Information PDF for details) (AVI)

Raw experimental video (AVI)

\section{AUTHOR INFORMATION}

\section{Corresponding Author}

*E-mail: zhengxu@lnm.imech.ac.cn.

\section{Notes}

The authors declare no competing financial interest.

\section{ACKNOWLEDGMENTS}

The authors acknowledge the National Natural Science Foundation of China (11572335, 11272321, and 11202219) and the Ministry of Science and Technology (2011CB707604) for financial support. And we are grateful to the fruitful discussions with Dr. P-Y Jin. 


\section{REFERENCES}

(1) Daumas, F.; Destainville, N.; Millot, C.; Lopez, A.; Dean, D.; Salomé, L. Confined Diffusion without Fences of a G-Protein-Coupled Receptor as Revealed by Single Particle Tracking. Biophys. J. 2003, 84, 356-366.

(2) Di Rienzo, C.; Piazza, V.; Gratton, E.; Beltram, F.; Cardarelli, F. Probing Short-Range Protein Brownian Motion in the Cytoplasm of Living Cells. Nat. Commun. 2014, 5, 5891.

(3) Soula, H.; Care, B.; Beslon, G.; Berry, H. Anomalous Versus Slowed-Down Brownian Diffusion in the Ligand-Binding Equilibrium. Biophys. J. 2013, 105, 2064-2073.

(4) Valentine, M. T.; Kaplan, P. D.; Thota, D.; Crocker, J. C.; Gisler, T.; Prud'homme, R. K.; Beck, M.; Weitz, D. A. Investigating the Microenvironments of Inhomogeneous Soft Materials with Multiple Particle Tracking. Phys. Rev. E: Stat. Phys., Plasmas, Fluids, Relat. Interdiscip. Top. 2001, 64, 061506.

(5) Skaug, M. J.; Wang, L.; Ding, Y.; Schwartz, D. K. Hindered Nanoparticle Diffusion and Void Accessibility in a Three-Dimensional Porous Medium. ACS Nano 2015, 9, 2148-2156.

(6) Mason, T. G.; Ganesan, K.; van Zanten, J. H.; Wirtz, D.; Kuo, S. C. Particle Tracking Microrheology of Complex Fluids. Phys. Rev. Lett. 1997, 79, 3282-3285.

(7) van Zanten, J. H.; Amin, S.; Abdala, A. A. Brownian Motion of Colloidal Spheres in Aqueous Peo Solutions. Macromolecules 2004, 37, 3874-3880.

(8) Wong, I. Y.; Gardel, M. L.; Reichman, D. R.; Weeks, E. R.; Valentine, M. T.; Bausch, A. R.; Weitz, D. A. Anomalous Diffusion Probes Microstructure Dynamics of Entangled F-Actin Networks. Phys. Rev. Lett. 2004, 92, 178101.

(9) Saxton, M. J. Anomalous Diffusion Due to Obstacles: A Monte Carlo Study. Biophys. J. 1994, 66, 394-401.

(10) Hofling, F.; Franosch, T. Anomalous Transport in the Crowded World of Biological Cells. Rep. Prog. Phys. 2013, 76, 046602.

(11) Wang, B.; Kuo, J.; Granick, S. Bursts of Active Transport in Living Cells. Phys. Rev. Lett. 2013, 111, 208102.

(12) Zheng, X.; ten Hagen, B.; Kaiser, A.; Wu, M.; Cui, H.; Silber-Li, Z.; Löwen, H. Non-Gaussian Statistics for the Motion of SelfPropelled Janus Particles: Experiment Versus Theory. Phys. Rev. E 2013, 88, 032304.

(13) Piskorz, T. K.; Ochab-Marcinek, A. A Universal Model of Restricted Diffusion for Fluorescence Correlation Spectroscopy. J. Phys. Chem. B 2014, 118, 4906-4912.

(14) Lushnikov, P. M.; Šulc, P.; Turitsyn, K. S. Non-Gaussianity in Single-Particle Tracking: Use of Kurtosis to Learn the Characteristics of a Cage-Type Potential. Phys. Rev. E 2012, 85, 051905.

(15) Wang, B.; Anthony, S. M.; Bae, S. C.; Granick, S. Anomalous yet Brownian. Proc. Natl. Acad. Sci. U. S. A. 2009, 106, 15160-15164.

(16) Wang, B.; Kuo, J.; Bae, S. C.; Granick, S. When Brownian Diffusion Is Not Gaussian. Nat. Mater. 2012, 11, 481-485.

(17) Kwon, G.; Sung, B. J.; Yethiraj, A. Dynamics in Crowded Environments: Is Non-Gaussian Brownian Diffusion Normal? J. Phys. Chem. B 2014, 118, 8128-8134.

(18) Phillies, G. D. J. In Complex Fluids the Gaussian Diffusion Approximation Is Generally Invalid. Soft Matter 2015, 11, 580-586.

(19) Metzler, R.; Jeon, J. H.; Cherstvy, A. G.; Barkai, E. Anomalous Diffusion Models and Their Properties: Non-Stationarity, NonErgodicity, and Ageing at the Centenary of Single Particle Tracking. Phys. Chem. Chem. Phys. 2014, 16, 24128-24164.

(20) Chubynsky, M. V.; Slater, G. W. Diffusing Diffusivity: A Model for Anomalous, yet Brownian, Diffusion. Phys. Rev. Lett. 2014, 113, 098302.

(21) Ernst, D.; Kohler, J.; Weiss, M. Probing the Type of Anomalous Diffusion with Single-Particle Tracking. Phys. Chem. Chem. Phys. 2014, 16, 7686-7691.

(22) Godec, A.; Bauer, M.; Metzler, R. Collective Dynamics Effect Transient Subdiffusion of Inert Tracers in Flexible Gel Networks. New J. Phys. 2014, 16, 092002.
(23) Ghosh, S. K.; Cherstvy, A. G.; Metzler, R. Non-Universal Tracer Diffusion in Crowded Media of Non-Inert Obstacles. Phys. Chem. Chem. Phys. 2015, 17, 1847-1858.

(24) Kim, J.; Kim, C.; Sung, B. J. Simulation Study of Seemingly Fickian but Heterogeneous Dynamics of Two Dimensional Colloids. Phys. Rev. Lett. 2013, 110, 047801.

(25) Massignan, P.; Manzo, C.; Torreno-Pina, J. A.; Garcia-Parajo, M. F.; Lewenstein, M.; Lapeyre, G. J., Jr. Nonergodic Subdiffusion from Brownian Motion in an Inhomogeneous Medium. Phys. Rev. Lett. 2014, 112, 150603.

(26) Szymanski, J.; Weiss, M. Elucidating the Origin of Anomalous Diffusion in Crowded Fluids. Phys. Rev. Lett. 2009, 103, 038102.

(27) Sprakel, J.; van der Gucht, J.; Cohen Stuart, M. A.; Besseling, N. A. M. Rouse Dynamics of Colloids Bound to Polymer Networks. Phys. Rev. Lett. 2007, 99, 208301.

(28) Sarmiento-Gomez, E.; Santamaria-Holek, I.; Castillo, R. MeanSquare Displacement of Particles in Slightly Interconnected Polymer Networks. J. Phys. Chem. B 2014, 118, 1146-1158.

(29) Mason, T. G. Estimating the Viscoelastic Moduli of Complex Fluids Using the Generalized Stokes-Einstein Equation. Rheol. Acta 2000, 39, 371-378.

(30) Sprakel, J.; van der Gucht, J.; Stuart, M. A. C.; Besseling, N. A. M. Brownian Particles in Transient Polymer Networks. Phys. Rev. E 2008, 77, 061502.

(31) Cai, L. H.; Panyukov, S.; Rubinstein, M. Mobility of Spherical Probe Objects in Polymer Liquids. Macromolecules 2011, 44, 78537863.

(32) Kalathi, J. T.; Yamamoto, U.; Schweizer, K. S.; Grest, G. S.; Kumar, S. K. Nanoparticle Diffusion in Polymer Nanocomposites. Phys. Rev. Lett. 2014, 112, 108301.

(33) van der Gucht, J.; Besseling, N. A. M.; Knoben, W.; Bouteiller, L.; Cohen Stuart, M. A. Brownian Particles in Supramolecular Polymer Solutions. Phys. Rev. E: Stat. Phys., Plasmas, Fluids, Relat. Interdiscip. Top. 2003, 67, 051106

(34) Raikher, Y. L.; Rusakov, V. V.; Perzynski, R. Brownian Motion in a Viscoelastic Medium Modelled by a Jeffreys Fluid. Soft Matter 2013, 9, 10857-10865.

(35) Ziębacz, N.; Wieczorek, S. A.; Kalwarczyk, T.; Fiałkowski, M.; Holyst, R. Crossover Regime for the Diffusion of Nanoparticles in Polyethylene Glycol Solutions: Influence of the Depletion Layer. Soft Matter 2011, 7, 7181-7186.

(36) Ochab-Marcinek, A.; Holyst, R. Scale-Dependent Diffusion of Spheres in Solutions of Flexible and Rigid Polymers: Mean Square Displacement and Autocorrelation Function for Fcs and Dls Measurements. Soft Matter 2011, 7, 7366-7374.

(37) Skaug, M. J.; Mabry, J.; Schwartz, D. K. Intermittent Molecular Hopping at the Solid-Liquid Interface. Phys. Rev. Lett. 2013, 110, 256101.

(38) Cai, L. H.; Panyukov, S.; Rubinstein, M. Hopping Diffusion of Nanoparticles in Polymer Matrices. Macromolecules 2015, 48, 847862.

(39) Guan, J.; Wang, B.; Granick, S. Even Hard-Sphere Colloidal Suspensions Display Fickian yet Non-Gaussian Diffusion. ACS Nano 2014, 8, 3331-3336.

(40) Wang, D.; He, C.; Stoykovich, M. P.; Schwartz, D. K. Nanoscale Topography Influences Polymer Surface Diffusion. ACS Nano 2015, 9, $1656-1664$

(41) Eldar, A.; Elowitz, M. B. Functional Roles for Noise in Genetic Circuits. Nature 2010, 467, 167-173.

(42) Chaudhuri, P.; Berthier, L.; Kob, W. Universal Nature of Particle Displacements Close to Glass and Jamming Transitions. Phys. Rev. Lett. 2007, 99, 060604.

(43) Charbonneau, P.; Jin, Y.; Parisi, G.; Zamponi, F. Hopping and the Stokes-Einstein Relation Breakdown in Simple Glass Formers. Proc. Natl. Acad. Sci. U. S. A. 2014, 111, 15025-15030.

(44) Kegel, W. K. Direct Observation of Dynamical Heterogeneities in Colloidal Hard-Sphere Suspensions. Science 2000, 287, 290-293.

(45) Magdziarz, M.; Weron, A.; Burnecki, K.; Klafter, J. Fractional Brownian Motion Versus the Continuous-Time Random Walk: A 
Simple Test for Subdiffusive Dynamics. Phys. Rev. Lett. 2009, 103, 180602. 